BMJ Open

Sport \&

Exercise

Medicine

\title{
Exploring the relationship between gut microbiota and exercise: short-chain fatty acids and their role in metabolism
}

\author{
Ryan A Carey, Doreen Montag
}

To cite: Carey RA, Montag D. Exploring the relationship between gut microbiota and exercise: short-chain fatty acids and their role in metabolism. BMJ Open Sport \& Exercise Medicine 2021;7:e000930. doi:10.1136/ bmjsem-2020-000930

Accepted 26 January 2021
Check for updates

(C) Author(s) (or their employer(s)) 2021. Re-use permitted under CC BY-NC. No commercial re-use. See rights and permissions. Published by BMJ.

Global Public Health, Queen Mary University of London, London, UK

Correspondence to Dr Doreen Montag; d.montag@qmul.ac.uk

\section{ABSTRACT}

The human body is host to a multitude of bacteria, fungi, viruses and other species in the intestine, collectively known as the microbiota. Dietary carbohydrates which bypass digestion and absorption are broken down and fermented by the microbiota to produce short-chain fatty acids (SCFAs). Previous research has established the role of SCFAs in the control of human metabolic pathways. In this review, we evaluate SCFAs as a metabolic regulator and how they might improve endurance performance in athletes. By looking at research conducted in animal models, we identify several pathways downstream of SCFAs, either directly modulating metabolic pathways through second messenger pathways or through neuronal pathways, that contribute to energy utilisation. These pathways contribute to efficient energy metabolism and are thus key to maximising substrate utilisation in endurance exercise. Future research may prove the usefulness of targeted dietary interventions allowing athletes to maximise their performance in competition.

\section{INTRODUCTION}

The human body is composed of a diverse array of microorganisms, including bacteria, archaea, viruses and fungi. ${ }^{1}$ These organisms are collectively known as the microbiota and reside throughout and on the body, including in the lungs, ${ }^{2}$ oral mucosa, ${ }^{3}$ uterus ${ }^{4}$ and on the skin. ${ }^{5}$ A key location for the microbiota is the gastrointestinal tract, in particular the large intestine, where it is calculated that there are up to 100 trillion $\left(1 \times 10^{14}\right)$ microbes. ${ }^{6}$ The role of the microbiota in human physiology is becoming increasingly understood, from its role in inflammatory diseases and hypersensitivity $^{7}$ to the importance of a diverse microbiota for mental health. ${ }^{8}$

The composition and function of the microbiota vary among different populations. This is particularly true of elite athletes and obese individuals compared with other human subjects. ${ }^{9}$ How the microbiota impacts body composition is understood to be through its influences on metabolic pathways (including liver and adipose tissue), in addition to energy

\section{Summary box}

What is already known

- Exercise conveys benefit for the health of gut bacteria.

- Regulation and refinement of host metabolism are necessary for exercise.

What are the new findings

- Short-chain fatty acids may be physiologically implicated in endurance exercise performance.

extraction from diet and hormone signalling in the gut. ${ }^{10}$

Additionally, in animal experiments, germfree (GF) mice were significantly worse at endurance exercise compared with mice that had Bacteroides fragilis colonisation of the colon. ${ }^{11}$ This poses the question: what contribution does the gut microbiota have on aerobic activity in athletes? One of the metabolites that may explain the role of the microbiota on exercise is short-chain fatty acids (SCFAs).

\section{Short-chain fatty acids}

Some dietary carbohydrates which bypass digestion are broken down and fermented by bacteria that make up the microbiota in the large intestine. These insoluble carbohydrates break down into three of the most common SCFAs-acetate, propionate and butyrate, composed of hydrocarbon chains of 2,3 and 4 carbon chains, respectively. Importantly, the amount of SCFAs is not only regulated by the type of bacteria, but also by food in the intestine. In that way, the composition and quantity of SCFAs can readily be modified through dietary interventions, making it a target for athletes. Our current understanding is SCFAs mediate the interaction between diet, microbiota and the host, primarily through activation of $\mathrm{G}$ proteincoupled receptors (GPCRs). ${ }^{12-14}$ 


\section{Fatty acid GPCRs}

SCFAs are not restricted to the lumen of the gut as they can be found systemically in the blood, pancreas and brain. ${ }^{15}$ GPR41 and GPR43 (also known as FFAR3 and FFAR2, respectively) are a small family of GPCRs of which the ligand is colonic SCFAs. ${ }^{16}$

There is evidence that the effects of SCFAs in health and disease are diverse, ${ }^{17-19}$ and the expression of these receptors throughout the body supports the hypothesis that SCFAs are essential in physiological signalling and control of metabolism throughout multiple organs. Therefore, regulation and refinement of host metabolism are necessary for exercise, in particular endurance exercise. $^{20}$

The recent paper by Frampton et $a l^{21}$ is an excellent review on SCFAs and metabolism. The authors review the wider scope of effects on skeletal muscle, such as the effect on muscle mass and muscle phenotype. Frampton et $a l^{21}$ analyse the indirect effects of SCFAs, such as the effect on inflammation, and the implications on skeletal muscle, but they do not go further to link this to aerobic performance. The authors review metabolic pathways, for example, carbohydrate metabolism without further explanation about how this would have an effect on skeletal muscle output, and importantly, without any mention of aerobic exercise performance. This recent review ${ }^{21}$ is in line with analysis of SCFAs in metabolism and cardiovascular health ${ }^{17}$ and in inflammation, glucose and lipid metabolism. $^{22}$

In contrast, our literature review is unique in the context of aerobic exercise performance, analysing the effects of SCFAs on aerobic capacity of skeletal muscle only.

\section{Exercise}

In professional athletes, and also amateur sporting populations, the ability to perform at consistently high levels during competition and training is vital. Endurance activities, such as marathon running, triathlons or crosscountry skiing, are multifaceted disciplines relying on effective and efficient energy metabolism.

SCFAs can be used directly as an additional substrate for metabolism. ${ }^{23}$ These are predominantly metabolised by enterocytes of the intestine and liver. ${ }^{24}$ SCFAs are independent of carnitine for uptake and result in intramitochondrial activation to acyl-CoA. ${ }^{25}$ They also act as dedicated signalling molecules modulating the metabolism of other substrates. ${ }^{15}$

While it has been shown that exercise conveys benefit for the health of gut bacteria, it has not been shown whether the converse is true-whether the health of the gut bacteria has an impact on performance. The exact role of the microbiota and the metabolic function and the effect of SCFAs in the body can provide a greater comprehension whether improving the health and diversity of the microbiota may provide a competitive advantage in athletes.
While the methodology of the included articles varies significantly, there are common themes in the research questions. First, we will explore the work by Arora et al, ${ }^{26}$ De Vadder et $a l^{27}$ and Korecka et $a l,{ }^{28}$ which investigate the effects of microbiota-derived metabolites in the enteroendocrine system. All three papers conducted experiments in murine models, except for Korecka et $a t^{28}$ who additionally used an in vitro cell line. The work by Kimura et $a l^{29}$ Wichmann et $a l^{30}$ and Zadeh-Tahmasebi et $a l^{31}$ explore the role of intestinal metabolites in the plasma and thus demonstrate to us the potential for system-wide effects of SCFAs. Finally, Barton et $a l^{32}$ the only study to use human subjects, corroborate earlier research by exploring the metabolic phenotyping and biodiversity of the microbiota in elite athletes. For an overview of the articles methods and key findings, please see the online supplemental appendix 1.

\section{The role of SCFAs in the enteroendocrine system}

Arora $e t a l^{26}$ set out to identify whether dietary supplementation of fermentable soluble flaxseed fibre affects SCFA production in mice colons and how the SCFAs from this fermentation and downstream activation of the GPR41 receptor affects the enteroendocrine function within the colon and ileum. The authors deduced the transcriptome of enteroendocrine cells in both the ileum and colon was upregulated when supplemented with flaxseed fibre in a mouse model, and this was under the control of SCFAs. The researchers identified that supplementing the mouse diet with flaxseed fibre, which in turn increased the availability of SCFAs, also observed that the transcriptome of enteroendocrine cells in both the ileum and colon was upregulated. Of note, genes associated with cell division and protein catabolism increased.

De Vadder et $a l^{27}$ demonstrate that intestinal metabolic pathways, in particular, upregulation of gluconeogenesis is, in part, under the control of SCFAs. First, the authors demonstrated, through Caco-2 cell models, that SCFAs could directly induce intestinal gluconeogenesis genes. As Caco-2 cells resemble a composition similar to the enterocytes of the small intestine, they were suitable for modelling. The authors showed that the 24-hour incubation with proprionate did not impact the expression of G6PC, PCK1 and MUT in Caco-2 cells. However, the appearance of G6PC and PCK1 was increased twofold to threefold in cells incubated with butyrate showing that butyrate, but not proprionate, is able to directly increase gene expression related to intestinal gluconeogenesis (p.67).

De Vadder et $a l^{27}$ then quantified intestinal glucose production in rat models, fed propionate, butyrate or fructo-oligosacharides (FOS), for 2 weeks. Resulting in glucose-specific activity being "lower in the portal vein than in the artery" (p.86), demonstrating that the intestine had released unlabelled glucose (synthesised through gluconeogenesis). The authors demonstrated also that $23 \%$ of all systematic glucose was derived from intestinal gluconeogenesis. 
De Vadder et $a l^{27}$ went on to show that proprionate was the greatest inducer of intestinal gluconeogenesis and that the mechanism of this was through a gut-brain communication mechanism leading to intestinal gluconeogenesis, rather than direct induction. Through capsacin-mediated periportal deafferentiation, the authors demonstrated that G6Pase activity and PCK1 protein expression were not increased in the intestine compared with controls. However, dietary butyrate and FOS did increase G6Pase activity and PCK1 expression in the treated rat group. This shows that there is a role of the periportal nervous system in transducing the effects of intestinal gluconeogenesis (and portal glucose sensing) from proprionate.

Unlike Arora $e t a l,{ }^{26}$ who demonstrated that there are localised effects in the gastrointestinal tract through gene expression, De Vadder et $a l^{27}$ show that localised control of metabolic pathways in the gastrointestinal tract is influenced through more comprehensive pathways, in particular a neuronal pathway connecting the gut and brain.

Korecka $e t a t^{28}$ explore the underlying mechanism of intestinal bacterial influencing metabolism, in particular by evaluating the role of angiopoietin-like protein 4 (ANGPTL4) in the gastrointestinal tract. The authors, using an in vitro cell model of three different cell lines, identified that ANGPTL4 is induced by the butyrate, and that can regulate host metabolism independent from PPAR- $\gamma$. They demonstrated butyrate could induce expression of ANGPTL4 mRNA in intestinal epithelial cells both in vitro and in vivo. They identified that when treating the cells with butyrate, the level of PPAR- $\gamma$ was lower. They also found that ANGPTL4 expression in the ileum and colonic cells increased when exposed to Clostridium tyrobutyricum bacteria, confirming the effect that bacteria-produced SCFAs has on the expression of ANGPTL4.

Korecka $e t a l^{28}$ further corroborate the role of SCFAs influencing metabolic pathways in the gastrointestinal tract. Unlike Arora et $a l^{26}$ and De Vadder et $a l^{27}$ who demonstrate that gastrointestinal, metabolic pathways are influenced by SCFAs through the GPR41 and GPR43 family of receptors, Korecka et $a l^{28}$ show that it is not just this family of receptors which contributes to metabolic changes, but the ANGPTL4 receptor too.

\section{System-wide effects of SCFAs}

Kimura $e t a l^{29}$ researched the role of the GPR43 (FFAR3) receptor in the regulation of energy balance, identifying the role of the receptor in insulin signalling in adipose tissue. The authors demonstrated that mice deficient in GPR43 receptors through gene knockout (GPR43-/-), become obese on a regular diet. However, by using promoter-driven transgenic mice that overexpressed GPR43 in adipose tissue, they identified that the mice remained lean even when fed a high-fat diet. They concluded that "GPR43 receptor acts as a sensor for excess dietary energy, thereby controlling body energy utilisation while maintaining metabolic homoeostasis" (p.2). The researchers identified in transgenic aP2-GPR43TG mice, overexpressing GPR43, that insulin sensitivity was improved. They identified insulin-induced Akt phosphorylation in white adipose tissue but not in muscle or liver tissue. Kimura $e t a l^{29}$ subsequently administered exogenous acetate and found this suppressed insulin signalling in adipocytes of wild-type mice. In the GPR43 deficient mice, on the other hand, this effect was unseen. They concluded, the activation of the GPR43 receptor by SCFAs supress insulin pathways in adipocytes, leading to the inhibition of fat accumulation in adipose tissue. The authors showed that the influence of SCFAs GPR43 receptors is wider than the gastrointestinal tract. They also demonstrated the importance of the GPR43 in the regulation of insulin gesturing in adipocytes. The delicate balance of storage versus catabolism of lipids is an essential regulator in host energy regulation and is essential for efficient use of energy substrates during exercise.

.Wichmann $e t a l^{30}$ looked at how the microbiota can modulate energy availability in the colon, through glucagon-like peptide 1 (GLP-1). Using mouse models, the authors compared portal vein levels of GLP-1 in GF mice to conventionally raised mice. The researchers identified that in mice that have an absence of intestinal microbiota, there is an upregulation of GLP-1 expression levels to slow intestinal emptying. Also observed was that the absence of microbially produced SCFAs in a GF mouse colon results in significantly higher plasma GLP-1 levels compared with conventionally raised mice.

Wichmann $e t a b^{30}$ measured caecal SCFA concentration as a measure of energy availability. They transplanted unfractionated microbiota from a conventionally raised donor into GF mice. Escherichia coli resulted in a small increase in acetate, but overall SCFAs were unchanged, as were an expression of Gcg and GLP-1 positive cells. Thus, it can be elucidated that the elevated GLP-1 expression is due to increased proglucagon gene expression in the colon. On the other hand, B. thetaiotaomicron levels resulted in increased acetate and propionate, a fourfold increase in total SCFAs, and an associated 2.5-fold decrease in Gcg and 1.7 decreases in GLP-1 positive cells. In sum, Wichmann et a $\vec{l}^{30}$ show that SCFA concentration in the gut modulates GLP-1, an important metabolic regulator in the plasma.

Using male Sprague-Dawley rats, Zadeh-Tahmasebi et $a \hat{l}^{31}$ conducted experiments to identify the metabolic changes in rodents exposed to SCFAs. The authors demonstrated that glucose production is modulated through a GPR43 negative feedback loop, dependent on neuronal networks, highlighting the role of intestinal metabolites in glucose homeostasis. To identify the underlying mechanism of propionate sensing, the researchers removed the ileal mucosa, lysed and subjected the tissue western blot analysis of GPR43. A positive result was identified, with GPR43 expression being shown in the ileal mucosal tissue. LV-FFAR shRNA was injected into ileum 
3 days before the clamp studies. They demonstrated that without GPR43 expression, glucose infusion rate and glucose production remained the same, showing that propionate sensing is dependent on the activation of GPR43.

The authors ${ }^{31}$ focused on identifying how the production of glucose is modulated by the gut bacteria and its metabolites. Similarly, Wichmann et $a \hat{l}^{0}$ and ZadehTahmasebi $e t a l^{31}$ identified the role of GLP-1, which regulates a neuronal network. This is not dissimilar to the work of De Vadder $e t a l^{27}$ who identified that localised control of metabolic pathways in the gastrointestinal tract is influenced through more extensive pathways, in particular a neuronal pathway connecting the gut and the brain. Zadeh-Tahmasebi $e t a l^{31}$ highlighted that neuronal networks influence glucose production.

\section{Relationship between microbiota and exercise}

Barton et $a \vec{l}^{32}$ analysed metabolic phenotyping and functional metagenomics of the gut microbiome of 40 international rugby union players compared with agematched healthy controls in two groups, body mass index (BMI) less than 25 and BMI greater than 25 to account for variability in body composition of rugby union players.

The diversity of microbiota was significantly higher in the athletes compared with both high BMI and low BMI human controls. In particular, there was an increase of the genus Akkermansia among the elite athletes. As a result of the increased biodiversity, the SCFA levels in faeces, as measured by "gas chromatography-mass spectrometry (GC-MS) showed significantly higher levels of acetate $(\mathrm{p}<0.001)$, propionate $(\mathrm{p}<0.001)$, butyrate $(p<0.001)$ and valerate $(p=0.011)$ in athletes relative to controls" (p.628). ${ }^{32}$ The authors accounted for dietary intake, primarily overall energy intake, sugar, starch, total carbohydrate, fat, protein and fibre intake. There was an important correlation between concentrations of propionate and protein intake. In addition, butyrate had a strong relation to the intake of dietary fibre.

Ninety-eight metabolic passageways differed between the three cohorts. In the elite athlete cohort, it was observed that there was the highest abundance of upregulated paths-29 out of 34 of these were involved in upregulation of metabolism. This included carbohydrate biosynthesis, cofactor biosynthesis and genes involved in energy metabolism. There was a statistically significant correlation between the metabolic pathways to GC-MSidentified faecal SCFA concentrations. Barton $e t a l^{32}$ were the only researchers who used humans as their experimental subjects. They corroborate work done in animal models, however, by showing upregulation of a significant number of genes associated with SCFAs.

Several bodies of research have identified that exercise has positive effects on the composition and diversity of the microbiota. ${ }^{33}$ In animal models, microbiota and associated metabolites influence metabolic pathways in the gastrointestinal tract, the adipose tissue and the metabolites, specifically SCFAs, are found systemically.
In this way, we can consider the possibility that SCFAs may provide an alternative fuel for endurance exercise through direct utilisation in the skeletal muscle and by liberating other carbohydrate substrates through modifying metabolism.

\section{Endurance exercise}

The demands of endurance exercise from a metabolic standpoint, particularly at elite levels, are significant. ${ }^{35}$ Liver glycogen supplements muscle glycogen as an energy reserve that can be used in prolonged exercise. To put this in perspective, the total body glycogen store is estimated to be $103 \mathrm{~mol}$ of adenosine triphosphate (ATP) at best, which is insufficient to provide the $150 \mathrm{~mol}$ of ATP needed for a marathon lasting $>2$ hours. ${ }^{36}$

It is well documented that after particularly longendurance events, there is a marked caloric deficit, ${ }^{37}$ depletion of muscle and liver glycogen stores and generation of ketone bodies. ${ }^{38}$ We can infer, then, that for maximal performance, metabolic flexibility is essential. ${ }^{39}$

It is known that SCFAs derived from the microbiota are directly used in skeletal muscle. ${ }^{40}$ First, the SCFA receptor GPR42 is expressed in muscle ${ }^{41}$ which suggests there may be a role for SCFA signalling within myocytes. However, there have been limited studies evaluating SCFAs on muscle metabolism. In experiments on obese rats, 6 months of acetate injections $(5.2 \mathrm{mg} / \mathrm{kg}$ of body weight) increased gene expression of oxidative phosphorylation and glucose metabolic pathways, including the genes encoding Glut4, myoglobin and AMPK. ${ }^{42}$

\section{SCFAs in the nervous system}

We have seen from the work of De Vadder et $a l^{27}$ that there is a gut-brain communication pathway reliant on SCFAs. Additional research has shown us that there are receptors for SCFAs throughout the nervous system. ${ }^{43}$ There is an expression of the SCFA receptor GPR41 in both autonomic and somatic sensory ganglia. This is particularly important in host metabolism in exercise, as a regulator of energy expenditure is the sympathetic nervous system. The identification of GPR41 receptors in the superior cervical ganglion suggests that GPR41 could be a candidate for an autonomic nervous system sensing of SCFAs originating from dietary fibres having a downstream effect on metabolism through nutrient sensing. ${ }^{43}$

Early research conducted in cultured superior cervical neurons showed us that GPR41 activation by SCFAs had been shown to release norepinephrine,$^{44}$ suggesting that SCFAs possess the ability to enhance sympathetic activity. This may be of significance in the context of exercise performance, as afferent neural information from muscles under load establishes a pattern of sympatheticoadrenal activity according to the relative intensity of the exercise. $^{45}$

\section{Metabolism}

The gut microbiota impacts nutrient intake and energy regulation and a microbiota dysbiosis can influence the 
development of obesity, insulin resistance and diabetes. ${ }^{44}$ In human subjects consuming a 'typical western diet', it was found that microbially produced SCFAs contributed around $10 \%$ of total energy requirements, and the contribution is expected to be higher for humans consuming high-fibre diets and for exclusively plant-eating species. ${ }^{46}$

\section{ANGPTL4 expression}

The angiopoietin-like proteins, a family of secreted proteins, have a role in energy metabolism. ${ }^{47}$ They are named because they share tertiary structural domains with angiopoietins. ${ }^{48}$ ANGPTL4 has been shown in previous research to be involved in upregulating lipoprotein lipase (LPL) activity, ${ }^{49}$ which is vital in metabolism, as LPL breaks down triglycerides into free fatty acids which can be used by metabolising tissue, through betaoxidation ${ }^{50}$ including in muscle tissue.

Korecka $e t a l^{28}$ showed that ANGPTL4 expression is induced by the SCFA butyrate in the gastrointestinal tract. While their work does not demonstrate to us whether butyrate-induced ANGPTL4 is available in skeletal muscle, other research by Catoire $e t a \tilde{l}^{1}$ concluded that during endurance exercise, induction of ANGPTL4 in non-exercising muscle reduces local fatty acid uptake, presumably to prevent fat overload, while directing fatty acids to the active skeletal muscle as fuel. Another limitation of the Korecka research was that butyrate was administered via oral gavage to GF mice. While this may show whether a form of exogenous SCFA may convey a benefit, an improved method could also be to increase SCFAs through modification of diet. Further research is warranted to identify whether the ANGPTL4 expressed by ileum and colonic epithelial cells has an impact on skeletal muscle tissue and to determine if effects are seen through a dietary modification to upregulate SCFAs.

\section{GLP-1 signalling}

The effect of GLP-1 on glucose metabolism is understood. ${ }^{52}$ However, exposure to SCFAs, through the work of Wichmann et $a l, l^{30}$ demonstrates that there are lower levels of GLP-1. Through the dogma of GLP-1 signalling in insulin secretion, we can deduce there is an associated decrease in insulin while glucagon increased. This resulted in increased glucose uptake in skeletal muscle and increased glucose production in the liver. These are key to increasing glucose availability for skeletal muscle and thus prolonging the time to exhaustion.

.The expression of the GPR41 receptor is present in enteroendocrine cells in the beta cells of the pancreas, ${ }^{53}$ which identifies that pancreatic release of insulin is not just under the control of circulating glucose and longchain fatty acid levels, but also under the influence of molecules from the colon. As such, these receptors are being considered as novel drug targets for the treatment of metabolic syndrome because they are activated by fatty acids. ${ }^{16}$ The role of SCFAs in glucose homeostasis in individuals without diabetes, in particular athletes, may be important for ensuring energy demands are met during competition.

\section{Implications for athletes}

In animal experiments, GF mice were shown to perform worse at endurance exercise than mice with $B$. fragilis colonisation of the colon. ${ }^{11}$ This corroborates the role of SCFAs in metabolic pathways. In animal models, there is a link between SCFAs, gene expression in the gut, ANGPTL4 expression and GLP-1 expression. Other research shows the role of intestinal metabolites in mitochondrial function. ${ }^{54}$ All such metabolic contributions show a promising avenue of research for how the gut can be fine-tuned in athletes to maximise performance. Furthermore, there may be an important cluster of pathways modulated by intestinal metabolites that thus far have been neglected. Optimum nutrition is essential for peak athlete performance, and we can see that going forward more research into specific dietary supplements that might target bacteria known to generate SCFAs and thus likely improve exercise performance is warranted..$^{55}$

\section{CONCLUSION}

The majority of research surrounding metabolites produced in the gastrointestinal tract has focused on outcomes concerning metabolic syndrome or other disease states. In this review, by concentrating on a relatively small number of articles that looked at pathways downstream of SCFAs, several have been identified that may be physiologically implicated in exercise performance. These data, when put together, show a highly promising avenue of research for how the gut can be fine-tuned in athletes to maximise performance in and outside competition. However, the lack of human trials or a consensus in methodology for exploring SCFA pathways is extremely limiting at this point. Furthermore, the lack of experimentation in athlete populations makes recommendations challenging and preliminary.

However, this is an exciting area of research that would benefit from further investigation. One possibility is to compare time with exhaustion in an animal model after exogenous SCFAs. Another could be to measure the plasma concentrations of SCFAs in athletes in competition to evaluate how performance is related.

Contributors Both authors have been conceiving the paper's methodology, which has been executed by the first author under the supervision of the corresponding author. The paper has been written and revised by both authors.

Funding The authors have not declared a specific grant for this research from any funding agency in the public, commercial or not-for-profit sectors.

Competing interests None declared.

Patient consent for publication Not required.

Provenance and peer review Not commissioned; externally peer reviewed.

Open access This is an open access article distributed in accordance with the Creative Commons Attribution Non Commercial (CC BY-NC 4.0) license, which permits others to distribute, remix, adapt, build upon this work non-commercially, and license their derivative works on different terms, provided the original work is properly cited, appropriate credit is given, any changes made indicated, and the use is non-commercial. See: http://creativecommons.org/licenses/by-nc/4.0/. 
ORCID iD

Doreen Montag http://orcid.org/0000-0003-1365-1913

\section{REFERENCES}

1 Kapitan M, Niemiec MJ, Steimle A. Fungi as part of the microbiota and interactions with intestinal bacteria. Fungal physiology and immunopathogenesis. Springer, 2018: 265-301.

2 Dickson RP, Erb-Downward JR, Martinez FJ, et al. The microbiome and the respiratory tract. Annu Rev Physiol 2016;78:481-504.

3 Gao L, Xu T, Huang G, et al. Oral microbiomes: more and more importance in oral cavity and whole body. Protein Cell 2018;9:488-500.

4 Baker JM, Chase DM, Herbst-Kralovetz MM. Uterine microbiota: residents, tourists, or invaders? Front Immunol 2018;9:208.

5 Chen $\mathrm{CH}$, Yorgason E. Redefining Asian masculinity in the age of global media. Asian Communication Research 2018;15:123-53.

6 Qin J, Li R, Raes J, et al. A human gut microbial gene catalogue established by metagenomic sequencing. Nature 2010;464:59-65.

7 Forbes JD, Azad MB, Vehling L, et al. Association of exposure to formula in the hospital and subsequent infant feeding practices with gut microbiota and risk of overweight in the first year of life. JAMA Pediatr 2018;172:e181161.

8 Winter G, Hart RA, Charlesworth RPG, et al. Gut microbiome and depression: what we know and what we need to know. Rev Neurosci 2018;29:629-43.

9 Tremaroli V, Bäckhed F. Functional interactions between the gut microbiota and host metabolism. Nature 2012;489:242-9.

10 Vrieze A, Holleman F, Zoetendal EG, et al. The environment within: how gut microbiota may influence metabolism and body composition. Diabetologia 2010;53:606-13.

11 Hsu YJ, Chiu CC, Li YP, et al. Effect of intestinal microbiota on exercise performance in mice. J Strength Cond Res 2015;29:552-8.

12 Louis P, Hold GL, Flint HJ. The gut microbiota, bacterial metabolites and colorectal cancer. Nat Rev Microbiol 2014;12:661-72.

13 Reichardt N, Duncan SH, Young P, et al. Phylogenetic distribution of three pathways for propionate production within the human gut microbiota. Isme J 2014;8:1323-35.

14 Ze X, Duncan SH, Louis P, et al. Ruminococcus bromii is a keystone species for the degradation of resistant starch in the human colon. Isme J 2012;6:1535-43.

15 Morrison DJ, Preston T. Formation of short chain fatty acids by the gut microbiota and their impact on human metabolism. Gut Microbes 2016;7:189-200.

16 Ichimura A, Hasegawa S, Kasubuchi M, et al. Free fatty acid receptors as therapeutic targets for the treatment of diabetes. Front Pharmacol 2014;5:236.

17 Chambers ES, Preston T, Frost G, et al. Role of gut microbiotagenerated short-chain fatty acids in metabolic and cardiovascular health. Curr Nutr Rep 2018;7:198-206.

18 Coggan AR, Coyle EF. Carbohydrate ingestion during prolonged exercise: effects on metabolism and performance. Exerc Sport Sci Rev 1991;19:1-40.

19 Melbye P, Olsson A, Hansen TH, et al. Short-Chain fatty acids and gut microbiota in multiple sclerosis. Acta Neurol Scand 2019:139:208-19.

20 Laursen PB, Rhodes EC. Factors affecting performance in an ultraendurance triathlon. Sports Med 2001;31:195-209.

21 Frampton J, Murphy KG, Frost G, et al. Short-Chain fatty acids as potential regulators of skeletal muscle metabolism and function. Nat Metab 2020;2:840-8.

$22 \mathrm{He} \mathrm{J}$, Zhang P, Shen L, et al. Short-Chain fatty acids and their association with signalling pathways in inflammation, glucose and lipid metabolism. Int J Mol Sci 2020;21:6356.

23 LeBlanc JG, Chain F, Martín R, et al. Beneficial effects on host energy metabolism of short-chain fatty acids and vitamins produced by commensal and probiotic bacteria. Microb Cell Fact 2017;16:1-10.

24 den Besten G, van Eunen K, Groen AK, et al. The role of short-chain fatty acids in the interplay between diet, gut microbiota, and host energy metabolism. J Lipid Res 2013;54:2325-40.

25 Schönfeld P, Wojtczak L. Short- and medium-chain fatty acids in energy metabolism: the cellular perspective. J Lipid Res 2016;57:943-54

26 Arora T, Rudenko O, Egerod KL, et al. Microbial fermentation of flaxseed fibers modulates the transcriptome of GPR41-expressing enteroendocrine cells and protects mice against diet-induced obesity. Am J Physiol Endocrinol Metab 2019;316:E453-63.

27 De Vadder F, Kovatcheva-Datchary P, Goncalves D, et al. Microbiotagenerated metabolites promote metabolic benefits via gut-brain neural circuits. Cell 2014;156:84-96.
28 Korecka A, de Wouters T, Cultrone A, et al. ANGPTL4 expression induced by butyrate and rosiglitazone in human intestinal epithelial cells utilizes independent pathways. Am J Physiol Gastrointest Liver Physiol 2013;304:G1025-37.

29 Kimura I, Ozawa K, Inoue D, et al. The gut microbiota suppresses insulin-mediated fat accumulation via the short-chain fatty acid receptor GPR43. Nat Commun 2013;4:1-12.

30 Wichmann A, Allahyar A, Greiner TU, et al. Microbial modulation of energy availability in the colon regulates intestinal transit. Cell Host Microbe 2013;14:582-90.

31 Zadeh-Tahmasebi M, Duca FA, Rasmussen BA, et al. Activation of short and long chain fatty acid sensing machinery in the ileum lowers glucose production in vivo. J Biol Chem 2016;291:8816-24.

32 Barton W, Penney NC, Cronin O, et al. The microbiome of professional athletes differs from that of more sedentary subjects in composition and particularly at the functional metabolic level. Gut 2018;67:625-33.

33 Allen JM, Mailing LJ, Niemiro GM, et al. Exercise alters gut microbiota composition and function in lean and obese humans. Med Sci Sports Exerc 2018;50:747-57.

34 Zhao X, Zhang Z, Hu B, et al. Response of gut microbiota to metabolite changes induced by endurance exercise. Front Microbiol 2018;9:765

35 Joyner MJ, Coyle EF. Endurance exercise performance: the physiology of champions. J Physiol 2008;586:35-44.

36 Berg JM, Tymoczko JL, Stryer LFreeman WH, ed. Biochemistry. Fifth Edition, 2002.

37 Barrero A, Erola P, Bescós R. Energy balance of triathletes during an ultra-endurance event. Nutrients 2015;7:209-22.

38 Ma S, Suzuki K. Keto-adaptation and endurance exercise capacity, fatigue recovery, and exercise-induced muscle and organ damage prevention: a narrative review. Sports 2019;7:40.

39 San-Millán I, Brooks GA. Assessment of metabolic flexibility by means of measuring blood lactate, fat, and carbohydrate oxidation responses to exercise in professional endurance athletes and less-fit individuals. Sports Med 2018;48:467-79.

40 McNabney SM, Henagan TM. Short chain fatty acids in the colon and peripheral tissues: a focus on butyrate, colon cancer, obesity and insulin resistance. Nutrients 2017:9:1348.

41 Brown AJ, Goldsworthy SM, Barnes AA, et al. The orphan G protein-coupled receptors GPR41 and GPR43 are activated by propionate and other short chain carboxylic acids. J Biol Chem 2003;278:11312-9.

42 Yamashita $\mathrm{H}$, Maruta $\mathrm{H}$, Jozuka $\mathrm{M}$, et al. Effects of acetate on lipid metabolism in muscles and adipose tissues of type 2 diabetic Otsuka Long-Evans Tokushima fatty (OLETF) rats. Biosci Biotechnol Biochem 2009;73:570-6.

43 Nøhr MK, Egerod KL, Christiansen SH, et al. Expression of the short chain fatty acid receptor GPR41/FFAR3 in autonomic and somatic sensory ganglia. Neuroscience 2015;290:126-37.

44 Kimura I, Inoue D, Maeda T, et al. Short-Chain fatty acids and ketones directly regulate sympathetic nervous system via $G$ protein-coupled receptor 41 (GPR41). Proc Natl Acad Sci U S A 2011;108:8030-5.

45 Conway J, Wheeler R, Sannerstedt R. Sympathetic nervous activity during exercise in relation to age. Cardiovasc Res 1971;5:577-81.

46 Bergman EN. Energy contributions of volatile fatty acids from the gastrointestinal tract in various species. Physiol Rev 1990;70:567-90

47 Tjeerdema N, Georgiadi A, Jonker JT, et al. Inflammation increases plasma angiopoietin-like protein 4 in patients with the metabolic syndrome and type 2 diabetes. BMJ Open Diabetes Res Care 2014;2:e000034.

48 Talmud PJ, Smart M, Presswood E, et al. ANGPTL4 E40K and T266M: effects on plasma triglyceride and HDL levels, postprandial responses, and CHD risk. Arterioscler Thromb Vasc Biol 2008;28:2319-25.

49 Dijk W, Kersten S. Regulation of lipoprotein lipase by ANGPTL4. Trends Endocrinol Metab 2014;25:146-55.

50 Goldberg IJ, Eckel RH, Abumrad NA. Regulation of fatty acid uptake into tissues: lipoprotein lipase- and CD36-mediated pathways. J Lipid Res 2009;50 Suppl:S86-90.

51 Catoire M, Alex S, Paraskevopulos N, et al. Fatty acid-inducible ANGPTL4 governs lipid metabolic response to exercise. Proc Natl Acad Sci U S A 2014:111:E1043-52.

52 Carlessi R, Chen Y, Rowlands J, et al. GLP-1 receptor signalling promotes $\beta$-cell glucose metabolism via mTOR-dependent HIF-1 $\alpha$ activation. Sci Rep 2017;7:1-13.

53 Veprik A, Laufer D, Weiss S, et al. GPR41 modulates insulin secretion and gene expression in pancreatic $\beta$-cells and modifies metabolic homeostasis in fed and fasting states. Faseb J 2016;30:3860-9. 
54 Clark A, Mach N. Exercise-Induced stress behavior, gut-microbiotabrain axis and diet: a systematic review for athletes. J Int Soc Sports Nutr 2016;13:43.
55 Simpson HL, Campbell BJ. Review article: dietary fibre-microbiota interactions. Aliment Pharmacol Ther 2015;42:158-79. 